Research Article

\title{
Flocking for Multiagent Systems with Partial Information Exchange Based on Inclusion Principle
}

\author{
Hui Wei (iD) and Xuebo Chen (iD) \\ School of Electronic and Information Engineering, University of Science and Technology Liaoning, Anshan 114051, China \\ Correspondence should be addressed to Xuebo Chen; xuebochen@126.com
}

Received 20 April 2020; Revised 14 June 2020; Accepted 20 June 2020; Published 18 July 2020

Academic Editor: Luis Payá

Copyright (C) 2020 Hui Wei and Xuebo Chen. This is an open access article distributed under the Creative Commons Attribution License, which permits unrestricted use, distribution, and reproduction in any medium, provided the original work is properly cited.

This paper investigates the flocking problem of multi-agents with partial information exchange, which means that only part, but not all, of the agents are informed of the group objective. A distributed flocking model based on the inclusion principle is provided to simplify the design and analysis of multi-agent systems. Furthermore, to reduce the communication energy consumption, an improved flocking algorithm based on the model is proposed to achieve stable flocking for all the agents. The stability of the multi-agent system is then established, with the help of the Lyapunov stability theorem and LaSalle's invariance principle. Especially, considering the individual heterogeneity in both nature and engineering applications, we also investigate the flocking problem of multi-agents with different sensing radiuses and equilibrium distances. Finally, two kinds of simulation results are presented to demonstrate the validity of the proposed results. This work provides an insight not only into the properties of the different species of individual flocking, but also into the theoretical framework for the engineering design of multi-agent systems considering individual heterogeneity.

\section{Introduction}

Flocking, a common phenomenon in nature, can eventually achieve a group objective through local communication among the neighbouring agents. Examples of these agents include flocks of birds, schools of fish, groups of ants, and crowds of people. For many decades, flocking problems have received a great deal of attention from researchers in biology, social science, physics, control science, computer science, and so on [1-10], due to the emergence of swarming for a group of agents with local interactions. This phenomenon of swarming finds a broad range of applications in swarm robots [11], mobile sensor networks [12], and unmanned aircraft systems (UAS) [13-15].

In 1986, Reynolds produced a computer model by simulating flocks of birds, which consists of three basic rules of cohesion, separation, and alignment [9]. Soon after this, Vicsek et al. first studied the collective behavior of bird swarms from a theoretical perspective [16-19]. For instance, Vicsek et al. [16] proposed a self-driven particle model in 1995, which mainly concentrated on the emergence of flocking, whereas Toner and $\mathrm{Tu}$ [17] preferred the continuum mechanics method. From the particle-based model proposed by Vicsek et al. [16], Helbing et al. [18] conducted a series of experiments to explore the mechanism of escape panic. In 2003, Jadbabaie et al. [19] studied the linear Vicsek model without noise and were the first to provide a theoretical explanation and mathematical proof of this model. In 2006, inspired by the Reynolds rules, Olfati-Saber [7] designed two typical flocking algorithms: flocking in an obstacle-free environment and flocking with obstacle avoidance. Moreover, he performed 2D flocking in the presence and absence of obstacles and 3D flocking in free space. In his algorithms, it was assumed that all the agents can obtain the information of the group objective (or virtual leader). Such an assumption, however, requires significant energy consumption during the flocking process. This makes it almost impossible to be implemented in engineering applications, such as multitarget consensus circle pursuit for multiagent systems via a distributed multiflocking method. To overcome this limitation, $\mathrm{Su}$ et al. [20] extended the typical flocking algorithm, considering that only partial agents are informed of the group objective, and then, numerical simulations indicated that most agents will asymptotically track the group objective, 
even if only a fraction of the group are informed agents. However, there are some uninformed agents that still fail to track the group objective during the flocking process. Therefore, it is necessary to improve the typical flocking algorithm further in this work.

The aforementioned works have greatly promoted the research process of flocking control for multi-agent systems and established an important foundation for future researches.

Recently, some studies were conducted that are closely related to the research topic of this paper. For example, Luo et al. [21] investigated the multi-target tracking problem of multi-agent systems, which, however, is still a deadlock problem. In order to overcome this problem, Pei et al. [22] proposed a local multi-flocking algorithm, which successfully resulted in the multi-target consensus pursuit for multi-agent systems. Xi et al. [23] provided a theoretical framework for the analysis of the output consensus problem of high-order linear time-invariant multi-agent systems, during the flocking process. Cao and Ren [24] presented a distributed consensus tracking algorithm for first-order kinematics and a distributed swarm tracking algorithm for second-order dynamics, which mainly concentrated on solving the distributed coordinated tracking problem. Based on the continuous-time information-weighted Kalman consensus filter (IWKCF), Luo and Li [25] proposed a distributed topology optimization scheme to decrease the communication complexity of flocking for multi-agent systems, in which each agent produces a local optimally rigid graph with its neighbouring agents. However, most of the above studies have merely focused on the homogeneous multi-agent system. Considering the individual characteristics, multi-agent systems can be divided into two types: homogeneous and heterogeneous [26]. Individual heterogeneity exists widely in both nature and engineering applications, such as multi-UAS in military confrontations, different growth backgrounds, social distancing, and economic strength in human society. Therefore, it is of great theoretical and practical significance for the research of the flocking problem of multi-agents with different equilibrium distances and sensing radiuses. Note that the equilibrium distance is proportional to the sensing radius (according to the works of [7]). For the sake of brevity, we will discuss only the different equilibrium distances later.

As further research is being conducted on multi-agent systems, we can determine that the motion consensus of multi-agents is similar to the synchronization problem of complex networks in essence, which is the solution to achieve the consistency of the state of all agents [27-29]. Furthermore, the inclusion principle and its extended pairwise decomposition can take full advantage of the interconnection relationship between subsystems, which have been widely applied in fields such as multi-area interconnected power systems [30-32], formation control of unmanned aerial vehicles [33], Petri nets [34], etc. Hence, the inclusion principle and its extended pair-wise decomposition can be chosen as a proper tool to simplify the design and analysis of flocking for multi-agent systems.

In this paper, we first divide a complex swarm system into multiple pair-wise subsystems based on the inclusion principle. Then, from the Lennard-Jones potential function [35] and self-organization process [36], an improved flocking algorithm is designed for each subsystem. Thereby, flocking of multi-agents with partial information exchange is achieved via the coordinated control of the pair-wise subsystems, which means that only a part, but not all, of the agents can be informed of the group objective. Compared with the typical flocking algorithm [7] and the existing distributed topology optimization scheme [25], this work, to some extent, has decreased the calculated amount and greatly reduces the communication energy consumption during the flocking process. Furthermore, considering the individual heterogeneity in both nature and engineering applications, the multi-agent system can be divided into three different species, according to the size of the equilibrium distance. Particularly, one of the aspects we are very interested in is the collision avoidance between different species of multi-agents during the flocking process. In order to explore the internal mechanism of this interesting problem, two kinds of simulations are designed to investigate the flocking of multi-agents with different equilibrium distances, which can provide an insight into the properties of the different species of individual flocking.

In brief, the main contributions of this paper are as follows: (1) A distributed flocking model based on the inclusion principle is proposed to simplify the design and analysis of flocking for multi-agent systems. (2) Based on the Lennard-Jones potential function and self-organization process, an improved flocking algorithm is designed to achieve flocking of multi-agents with partial information exchange. (3) Considering the individual heterogeneity in both nature and engineering applications, the flocking problem of multi-agents with different equilibrium distances is also investigated.

An outline of the paper is organized as follows. Section 2 introduces some preliminary knowledge. A distributed flocking model based on the inclusion principle is presented in Section 3. Section 4 proposes an improved flocking algorithm. In Section 5, the stability analysis of multi-agent systems is proven with the help of the Lyapunov stability theorem and LaSalle's invariance principle. Several simulation results are provided in Section 6. Finally, the conclusions are presented in Section 7.

\section{Preliminaries}

The flocking algorithm design process proposed in this paper is based on some preliminary knowledge about graph theory, especially, the inclusion principle and its extended pairwise decomposition.

2.1. Graph Theory. For the description of multi-agent systems by graph theory in this paper, we have some definitions as follows [37].

Definition 1. Suppose that a network topology consists of $N$ vertexes. The network topology can be described by an undirected graph $G=(v, E)$, where $v=\{1,2, \ldots, N\}$ is a 
vertex set and $E \subseteq\{(i, j): i, j \in v, j \neq i\}$ denotes the edge set with vertexes of junctions.

Definition 2. The adjacency matrix $A_{a}=\left[a_{i j}\right]$ of the undirected graph $G$ is defined as

$$
a_{i j}= \begin{cases}1, & \text { if }(i, j) \in v, \\ 0, & \text { others, }\end{cases}
$$

where $A_{a}$ is symmetric, i.e. $A_{a}^{T}=A_{a}$.

Definition 3. The degree matrix of undirected graph $G$ is a diagonal matrix $D=D\left(A_{a}\right)$ with diagonal elements $\sum_{j=1}^{n} a_{i j}$ that are row sums of $A_{a}$. The Laplacian matrix $L=\left[l_{i j}\right]$ is an $n \times n$ matrix, which is defined as

$$
L=D-A_{a} .
$$

2.2. Inclusion Principle. As mentioned in Section 1, the inclusion principle is a theoretical basis for pair-wise decomposition and decentralized control of complex interconnected systems [32]. In order to control multi-agent systems coordinately, we introduce some definitions and theorems as follows [30].

Consider a continuous-time linear time-invariant system $S$ and its expanded system $\widetilde{S}$ described by

$$
\begin{array}{ll}
\mathbf{S}: \dot{x}=A x+B u, & y=C x, \\
\widetilde{\mathbf{S}}: \dot{\tilde{x}}=\widetilde{A} \widetilde{x}+\widetilde{B} \widetilde{u}, & \widetilde{y}=\widetilde{C} \widetilde{x},
\end{array}
$$

where $x \in R^{n}, u \in R^{m}$, and $y \in R^{l}$ are the state, input, and output vectors of the system $S$, respectively. $\widetilde{x}(t) \in R^{\tilde{n}}$, $\widetilde{u}(t) \in R^{\tilde{m}}$, and $\tilde{y}(t) \in R^{l}$ are the state, input, and output vectors of the expanded system $\widetilde{\mathbf{S}}$, respectively. The matrices $A, B, C$ and $\widetilde{A}, \widetilde{B}, \widetilde{C}$ have appropriate dimensions. It is supposed that $n \leq \widetilde{n}, m \leq \widetilde{m}$, and $l \leq \widetilde{l}$.

Definition 4. The expanded system $\widetilde{\mathbf{S}}$ includes the system $\mathbf{S}$, namely, $\widetilde{\mathbf{S}}^{S}$. If there is a quadruplet of full-rank matrices $\{V, U, R, S\}$ satisfying $U V=I_{n}$, such that for any initial condition $x_{0} \in R^{n}$ and any input $u \in R^{m}$, when $\tilde{x}_{0}=V x_{0}$, $\tilde{u}=R u$, there is $x\left(t ; t_{0}, x_{0}, u\right)=U \widetilde{x}\left(t ; t_{0}, \tilde{x}_{0}, \tilde{u}\right)$ and $y[x(t)]=S \widetilde{y}[\tilde{x}(t)]$ for all $t \geq t_{0}$.

Theorem 1. If the system $S$ is a restriction of the expanded system $\widetilde{S}$, namely $S \subset{ }^{R} \widetilde{S}$, then there exists a triplet of full-rank matrices $\{V, R, T\}$ such that

$$
\begin{aligned}
\widetilde{A} V & =V A, \widetilde{B} R=V B, \widetilde{C} V=T C, \\
\text { or } M_{A} V & =0, M_{B} R=0, M_{C} V=0,
\end{aligned}
$$

where $V, R, T, M_{A}, M_{B}$, and $M_{B}$ will be illustrated in Theorem 2.

Proof. The detailed proof is carried out in [30].

Theorem 2. If the system $S$ is an aggregation of the expanded system $\widetilde{S}$, namely, $S \subset{ }^{A} \widetilde{S}$, then there exists a triplet of fullrank matrices $\{U, Q, S\}$ such that

$$
\begin{aligned}
U \widetilde{A} & =A U, U \widetilde{B}=B Q, S \widetilde{C}=C U, \\
\operatorname{or} U M_{A} & =0, U M_{B} R=0, S M_{C} V=0 .
\end{aligned}
$$

As mentioned above, the relationship between the system $\mathbf{S}$ and expanded system $\widetilde{\mathbf{S}}$ is described by

$$
\begin{gathered}
\widetilde{A}=V A U+M_{A}, \\
\widetilde{B}=V B Q+M_{B}, \\
\widetilde{C}=T C U+M_{C},
\end{gathered}
$$

where $V, U, R, Q, T$, and $S$ are full-rank transformation matrices with dimensions of $\widetilde{n} \times n, \widetilde{n} \times n, \widetilde{m} \times m, \widetilde{m} \times m$, and $\widetilde{l} \times l, \tilde{l} \times l$, respectively, and satisfy $U V=I_{n}, Q R=I_{m}$, and $S T=I_{l} . M_{A}, M_{B}$, and $M_{C}$ are complementary matrices with dimensions of $\widetilde{n} \times \widetilde{n}, \widetilde{m} \times \widetilde{m}$, and $\widetilde{l} \times \widetilde{l}$, respectively.

Proof. The detailed proof is carried out in [30].

The system $\mathbf{S}$ can be decomposed into various forms. A typical decomposition is nonoverlapped; the dynamic subsystems are identified as

$$
\mathbf{S}_{i j}: \dot{x}_{i}=A_{i i} x_{i}+B_{i i} u_{i}, y_{i}=C_{i i} x_{i}, \quad i=1,2, \ldots, N,
$$

where $x_{i} \in R^{n_{i}}, u_{i} \in R^{m_{i}}$, and $y_{i} \in R^{l_{i}}$ are the state, input, and output vectors of the subsystem $S_{i}$, respectively. Meanwhile, the decomposition is usually overlapped by some states, inputs, outputs, and even a subsystem between pair-wise subsystems. For example, pair-wise subsystems can be described by

$$
\begin{aligned}
\mathbf{S}_{i j}:\left[\begin{array}{c}
\dot{x}_{i} \\
\dot{x}_{j}
\end{array}\right] & =\left[\begin{array}{cc}
A_{i i} & A_{i j} \\
A_{j i} & A_{j j}
\end{array}\right]\left[\begin{array}{l}
x_{i} \\
x_{j}
\end{array}\right]+\left[\begin{array}{cc}
B_{i i} & 0 \\
0 & B_{j j}
\end{array}\right]\left[\begin{array}{l}
u_{i} \\
u_{j}
\end{array}\right], \\
{\left[\begin{array}{c}
y_{i} \\
y_{j}
\end{array}\right] } & =\left[\begin{array}{cc}
C_{i i} & 0 \\
0 & C_{j j}
\end{array}\right]\left[\begin{array}{c}
x_{i} \\
x_{j}
\end{array}\right], \\
i & =1,2, \ldots, N-1, \quad j=i+1, \ldots, N .
\end{aligned}
$$

Theorem 3. Let

$$
\widetilde{S}_{p}: \dot{\tilde{x}}_{p}=\widetilde{A}_{p} \widetilde{x}_{p}+\widetilde{B}_{p} \widetilde{u}_{p}, \tilde{y}_{p}=\widetilde{C}_{p} \widetilde{x}_{p},
$$

where $\tilde{x}_{p} \in R^{\tilde{n}}, \tilde{u}_{p} \in R^{\tilde{m}}$, and $\tilde{y}_{p} \in \tilde{R}^{\tilde{l}}$ are the state, input, and output vectors of the system $\widetilde{S}_{p}$, respectively. Suppose that the expanded system $\widetilde{S}$ includes the system $S$, namely, $\widetilde{S}$, and $\tilde{x}_{p}=P_{A}^{-1} \tilde{x}, \tilde{u}_{p}=P_{B}^{-1} \tilde{u}, \tilde{y}_{p}=P_{C}^{-1} \tilde{y}$, where $P_{A}, P_{B}$, and $P_{C}$ are nonsingular permutation matrices with appropriate dimensions, such that $\widetilde{A}_{P}=P_{A}^{-1} \widetilde{A} P_{A}, \widetilde{B}_{P}=P_{A}^{-1} \widetilde{B} P_{B}$, and $\widetilde{C}_{P}=P_{C}^{-1} \widetilde{C} P_{A}$. Hence, $\widetilde{S}_{P}^{S}$ satisfying $U_{P}=U P_{A}, V_{P}=P_{A}^{-1} V, R_{P}=P_{B}^{-1} R$, and $T_{P}=P_{C}^{-1} T$.

Proof. The detailed proof is carried out in [30].

\section{Distributed Flocking Model Based on Inclusion Principle}

During the flocking process of multi-agents, the dynamics of the multi-agent system should be modeled mathematically to facilitate further investigation. This does not depend on 
whether the agent is natural biology (e.g., bees and ants) or a piece of engineering equipment (e.g., robots and UAVs). In this section, we assume that all agents have the same mass and size. Consider a group of $N$ mobile agents (or subsystems) with second-order linear dynamics, which is described by

$$
\begin{aligned}
\mathbf{S}_{i}:\left[\begin{array}{c}
\dot{p}_{i} \\
\dot{v}_{i}
\end{array}\right] & =\left[\begin{array}{ll}
0 & 1 \\
0 & 0
\end{array}\right]\left[\begin{array}{l}
p_{i} \\
v_{i}
\end{array}\right]+\left[\begin{array}{l}
0 \\
1
\end{array}\right] u_{i}, \\
{\left[\begin{array}{c}
y_{p i} \\
y_{v i}
\end{array}\right] } & =\left[\begin{array}{ll}
1 & 0 \\
0 & 1
\end{array}\right]\left[\begin{array}{l}
p_{i} \\
v_{i}
\end{array}\right], \quad i=1,2, \ldots, N,
\end{aligned}
$$

where $p_{i}, v_{i} \in R^{m}$ (e.g., $m=2$ or 3 , when a flocking in a $2 \mathrm{D}$ or $3 \mathrm{D}$ space) denote the position and velocity vector of the agent $i$, respectively. $u_{i} \in R^{m}$ is the control input of agent $i$. Suppose that each agent has a limited sensing radius (or interaction range). Let $r>0$ denotes the sensing radius, and then, the neighbor set of the agent $i$ is defined as

$$
N_{i}=\left\{j \in v:\left\|p_{j}-p_{i}\right\|<r\right\}
$$

where $\|\cdot\|$ is the Euclidean norm in $R^{m}$; each agent can acquire the information of other mobile agents within its neighbor set. Moreover, one dynamic (or static) $\gamma$-agent represents the group objective (or virtual leader) of multiagent systems, which drives all agents to track the group objective with the following model:

$$
\left\{\begin{array}{l}
\dot{p}_{\gamma}=v_{\gamma} \\
\dot{v}_{\gamma}=f_{\gamma}\left(p_{\gamma}, v_{\gamma}\right)
\end{array}\right.
$$

where $p_{\gamma}, v_{\gamma}, f_{\gamma} \in R^{m}$ denote the position, velocity, and control input vector of the group objective, respectively. The initial state vector pairs are set to $\left(p_{\gamma}(0), v_{\gamma}(0)\right)=$ $\left(p_{d}, v_{d}\right)$. A static $\gamma-$ agent means that its state is fixed, that is, $\left(p_{\gamma}, v_{\gamma}\right)=\left(p_{d}, v_{d}\right)$ for all the way. In Section 6, we assume that the group objective always moves at a constant velocity to the fixed direction. Thereby, dynamic equation (12) of the group objective is simplified as $\dot{p}_{\gamma}=v_{d}$, $\dot{v}_{\gamma}=0$.

Considering that $p_{i}, v_{i}$ are measurable, the linear state-space model of the multi-agent system can be described by

$$
\mathrm{S}: \dot{x}=A x+B u, y=C x,
$$

where $\quad x=\left(p_{1}, v_{1}, p_{2}, v_{2}, \ldots, p_{N}, v_{N_{T}}\right)^{T}, \quad u=\left(u_{1}, u_{2}, \ldots\right.$, $\left.u_{N}\right)^{T}$, and $y=\left(y_{p_{1}}, y_{v_{1}}, \ldots, y_{p_{N}}, y_{v_{N}}\right)^{T}$ are the state, input, and output vectors of the system $\mathbf{S}$, respectively. Meanwhile,

$A=\left[\begin{array}{ccc}A_{11} & \cdots & A_{1 N} \\ \vdots & \ddots & \vdots \\ A_{N 1} & \cdots & A_{N N}\end{array}\right], B=\operatorname{blockdiag}\left[B_{11}, \ldots, B_{N N}\right]$, and $C=\operatorname{blockdiag}\left[C_{11}, \ldots, C_{N N}\right]$, in which $A_{i j}, B_{i i}$, and $C_{i i}$ are submatrices with dimensions of $n_{i} \times n_{j}, n_{i} \times m_{i}$, and $l_{i} \times n_{i}$, respectively, and $i=1,2, \ldots, N, j=1,2, \ldots, N$. According to equations (10), (11), and (13), pairwise subsystems (or neighbouring agents) can be identified by

$$
\begin{aligned}
\mathbf{S}_{i j}:\left[\begin{array}{c}
\dot{p}_{i} \\
\dot{v}_{i} \\
\dot{d}_{i j} \\
\dot{p}_{j} \\
\dot{v}_{j}
\end{array}\right] & =\left[\begin{array}{ccccc}
0 & 1 & 0 & 0 & 0 \\
0 & 0 & 0 & 0 & 0 \\
0 & -1 & 0 & 0 & 1 \\
0 & 0 & 0 & 0 & 1 \\
0 & 0 & 0 & 0 & 0
\end{array}\right]\left[\begin{array}{c}
p_{i} \\
v_{i} \\
d_{i j} \\
p_{j} \\
v_{j}
\end{array}\right]+\left[\begin{array}{ll}
0 & 0 \\
1 & 0 \\
0 & 0 \\
0 & 0 \\
0 & 1
\end{array}\right]\left[\begin{array}{l}
u_{i} \\
u_{j}
\end{array}\right], \\
{\left[\begin{array}{c}
y_{p_{i}} \\
y_{v_{i}} \\
y_{d_{i j}} \\
y_{p_{j}} \\
y_{v_{j}}
\end{array}\right] } & =\left[\begin{array}{lllll}
1 & 0 & 0 & 0 & 0 \\
0 & 1 & 0 & 0 & 0 \\
0 & 0 & 1 & 0 & 0 \\
0 & 0 & 0 & 1 & 0 \\
0 & 0 & 0 & 0 & 1
\end{array}\right]\left[\begin{array}{c}
p_{i} \\
v_{i} \\
d_{i j} \\
p_{j} \\
v_{j}
\end{array}\right], \\
i & =1,2, \ldots, N, \quad \forall j \in N_{i},
\end{aligned}
$$

where $\left\|d_{i j}\right\|=\left\|p_{j}-p_{i}\right\|$.

To illustrate the applicability of the pair-wise decomposition [30] and the improved flocking algorithm (see Section 4), we consider an interconnected multi-agent system (the number of agents $N \geq 3$ ) as an example. This system with its equilibrium state is shown in Figure 1(a). The vertices denote the subsystems (or agents), represented by blue spheres. The edges denote the interconnections between the neighbouring subsystems, represented by solid lines. The dashed ovals represent the pair-wise subsystems that can be decomposed, as shown in Figure 1(b). The basic interconnection coefficient $e_{i j}$ is used to represent the subsystems' connection states, if the subsystem $\mathbf{S}_{i}$ is connected with the subsystem $\mathbf{S}_{j}$, then $e_{i j}=1$; , otherwise, $e_{i j}=0$. From Figure 1(c), the dashed ovals, respectively, divide the pair-wise subsystem in the system $\mathbf{S}$, among which the intersections, namely, intersected subsystems, are the overlapped parts between subsystems, that are also called overlapped subsystems.

Based on the inclusion principle and its extended pairwise decomposition [32], we consider the coordinated control of each pair-wise subsystem as the basic control of the multi-agent system. Then, the flocking control for the whole system is realized through the parallel coordinated control of these pair-wise subsystems, thereby simplifying the analysis and design of such complex systems. For example, when $N=3$, the system (shown in Figure $1(\mathrm{~b})$ ) is represented by equation (13) with

$$
\begin{aligned}
A & =\left[\begin{array}{lll}
A_{11} & A_{12} & A_{13} \\
A_{21} & A_{22} & A_{23} \\
A_{31} & A_{32} & A_{33}
\end{array}\right], \\
B & =\text { blockdiag }\left[B_{11}, B_{22}, B_{33}\right], \\
C & =\text { blockdiag }\left[C_{11}, C_{22}, C_{33}\right]=I .
\end{aligned}
$$

The topology can be considered as a composite of the loop structure between the subsystems $\mathbf{S}_{1}, \mathbf{S}_{2}$, and $\mathbf{S}_{3}$. From Theorems 1 and 2, we apply the following expansion transformations: 


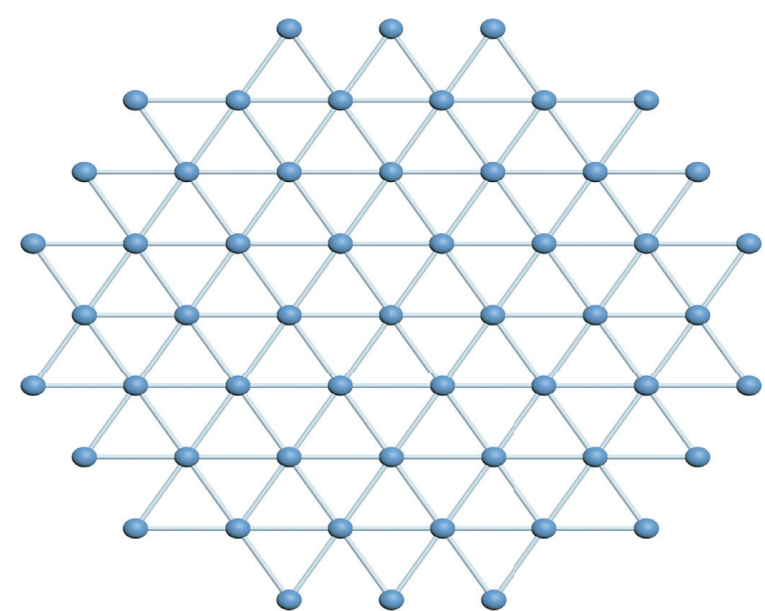

(a)

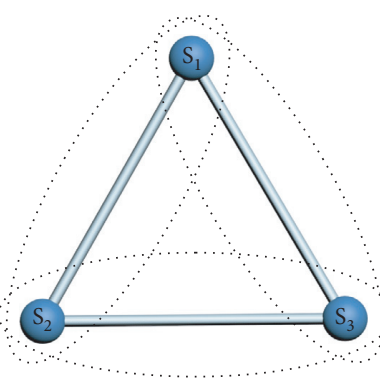

(b)

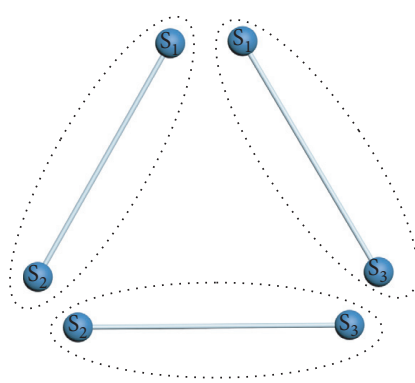

(c)

Figure 1: Schematic diagram of stable flocking for multiagent systems. (a) The whole system. (b) The system with $N=3$. (c) The expanded pairwise subsystems.

$$
\begin{gathered}
V=\operatorname{blockdiag}\left(\left[I_{11}, I_{11}\right]^{T},\left[I_{22}, I_{22}\right]^{T},\left[I_{33}, I_{33}\right]^{T}\right), \\
U=0.5 \text { blockdiag }\left(\left[I_{11}, I_{11}\right],\left[I_{22}, I_{22}\right],\left[I_{33}, I_{33}\right]\right), \\
M_{A}=0.5 \times\left[\begin{array}{cccccc}
A_{11} & -A_{11} & A_{12} & -A_{12} & -A_{13} & A_{13} \\
-A_{11} & A_{11} & A_{12} & -A_{12} & -A_{13} & A_{13} \\
-A_{21} & A_{21} & A_{22} & -A_{22} & 0 & 0 \\
-A_{21} & A_{21} & -A_{22} & A_{22} & 0 & 0 \\
A_{31} & -A_{31} & 0 & 0 & A_{33} & -A_{33} \\
A_{31} & -A_{31} & 0 & 0 & -A_{33} & A_{33}
\end{array}\right] .
\end{gathered}
$$

Hence,

$$
\widetilde{A}=\left[\begin{array}{cccccc}
A_{11} & 0 & A_{12} & 0 & 0 & A_{13} \\
0 & A_{11} & A_{12} & 0 & 0 & A_{13} \\
0 & A_{21} & A_{22} & 0 & 0 & 0 \\
0 & A_{21} & 0 & A_{22} & 0 & 0 \\
A_{31} & 0 & 0 & 0 & A_{33} & 0 \\
A_{31} & 0 & 0 & 0 & 0 & A_{33}
\end{array}\right] .
$$
matrix,

Then, according to Theorem 3, we use the permutation

$$
P_{A}=\prod_{k=1}^{5} p_{A k(k+1)}=p_{A 12} p_{A 23} p_{A 34} p_{A 45} p_{A 56}
$$

resulting in

$$
\widetilde{A}_{p}=p_{A}^{-1} \widetilde{A} P_{A}=\left[\begin{array}{cccccc}
A_{11} & A_{12} & 0 & 0 & A_{13} & 0 \\
A_{21} & A_{22} & 0 & 0 & 0 & 0 \\
0 & 0 & A_{22} & A_{23} & 0 & 0 \\
0 & 0 & A_{32} & A_{33} & 0 & 0 \\
0 & 0 & 0 & 0 & A_{33} & A_{31} \\
0 & A_{12} & 0 & 0 & A_{13} & A_{11}
\end{array}\right] \text {, }
$$

where we have the desired pair-wise control structure as shown in Figure 1(c), that is, each pair-wise subsystem satisfies equation (14).

According to equation (11), the equilibrium structure (or desired geometry) of flocking requires that the distance among the neighbouring agents is constant, thereby satisfying the following constraints:

$$
\left\|p_{j}-p_{i}\right\|=d_{e}
$$

where $i=1,2, \ldots, N, \forall j \in N_{i}(t)$ as time $t \longrightarrow \infty$. The equilibrium distance $d_{e}$ is a positive constant, usually $d_{e}<r$. Particularly, the equilibrium distance plays an important role in the desired formation (e.g., a geometric model of flocks).

\section{Flocking Control Algorithm}

From the above distributed flocking model, an improved flocking algorithm is designed to achieve stable flocking of multi-agents. Since 2006, the typical flocking algorithm, proposed by Olfati-Saber [7], has been extensively applied in fields such as mobile sensor networks and unmanned aircraft systems (UAS), etc. [12-15]. In [7], the control protocol (or input) $u_{i}$ for agent $i$ consists of the following three components:

$$
u_{i}=u_{i}^{g}+u_{i}^{d}+u_{i}^{\gamma}
$$

where $u_{i}^{g}$ and $u_{i}^{d}$ are the gradient-based term and velocity consensus term of the control protocol $u_{i}$, respectively. $u_{i}^{\gamma}$ is the navigational feedback to track the group objective, which is described by

$$
u_{i}^{\gamma}=c_{1}\left(p_{\gamma}-p_{i}\right)+c_{2}\left(v_{\gamma}-v_{i}\right), \quad c_{1}, c_{2}>0 .
$$

In this section, a novel distributed flocking algorithm is considered based on the Lennard-Jones potential function [35] and the self-organization process [36]. In contrast with the typical flocking algorithm [7], we simplify the interaction 
protocols between the neighbouring agents and develop a complex flocking of multi-agents, via a simpler pair-wise action function that only considers repulsion and attraction between the neighbouring agents (or the pair-wise subsystems). More specifically, according to (14) and (20), the control protocol $u_{i}$ for the agent $i$ (or the subsystem $\mathbf{S}_{i}$ ) is given by the following function:

$$
\begin{aligned}
u_{i}= & \sum_{j \in N_{i}} f_{\alpha}\left(\left\|p_{j}-p_{i}\right\|\right) e_{i j}+\sum_{j \in N_{i}} a_{i j}\left(v_{j}-v_{i}\right) \\
& +c_{1}\left(p_{\gamma}-p_{i}\right)+c_{2}\left(v_{\gamma}-v_{i}\right), \quad i \in v,
\end{aligned}
$$

where $e_{i j}=\left(\left(p_{j}-p_{i}\right) /\left(\sqrt{1+\varepsilon\left\|p_{j}-p_{i}\right\|^{2}}\right)\right), \varepsilon \in(0,1)$ is a value along the line connecting $p_{i}$ to $p_{j}$, and $a_{i j}$ is the element of the adjacency matrix $A_{a}$ as in Definition 2 and satisfies (1). In addition, compared with the traditional artificial potential function [7, 20-25], a simpler pair-wise action function that only considers repulsion and attraction among the neighbouring agents (or the pair-wise subsystems) is presented to reflect the interaction protocols. In this way, to some extent, the calculation difficulties can be decreased during the flocking process. Thus, the pair-wise action function $f_{\alpha}(x)$ is defined as

$$
f_{\alpha}(x)= \begin{cases}k_{1}\left(\frac{1}{x}-\frac{1}{d_{e}}\right), & x \leq d_{e}, \\ 0, & x>r, \\ k_{2}\left(d_{e}-x\right) e^{\left(\left(\left(x-d_{e}\right)^{2}\right) / r\right),} & d_{e}<x \leq r,\end{cases}
$$

where $k_{1}$ and $k_{2}$ are defined as the coefficients of the interconnection force, respectively, and especially, the pairwise action function $f_{\alpha}(x)$ can be drawn as in Figure 2 when $d_{e}=5$. Figure 2 shows that the action force between the neighbouring agents is an attraction for a long range, but repulsion for a short range. Moreover, the pair-wise potential function $F_{\alpha}(x)$ is described by

$$
F_{\alpha}(x)=\int_{d_{e}}^{x} f_{\alpha}(s) \mathrm{d} s .
$$

Note that the pair-wise potential function $F_{\alpha}\left(\left\|p_{j}-p_{i}\right\|\right)$ is a differential, nonnegative function satisfying the following conditions: (1) $F_{\alpha}$ reaches its unique minimum when $\left\|p_{j}-p_{i}\right\|=d_{e}$; (2) $F_{\alpha} \longrightarrow \infty$ as $\left\|p_{j}-p_{i}\right\| \longrightarrow 0$; (3) $F_{\alpha}$ remains constant if $\left\|p_{j}-p_{i}\right\|>r$.

In the flocking control protocol (23), it is assumed that all agents can obtain the information of the group objective, which is almost impossible and requires considerable energy consumption in practice. For the purpose of reducing the communication energy consumption, in this section, we assume that only a part, but not all, of the agents are informed of the group objective. Consequently, the control protocol (23) is modified as

$$
\begin{aligned}
u_{i}= & \sum_{j \in N_{i}} f_{\alpha}\left(\left\|p_{j}-p_{i}\right\|\right) e_{i j}+\sum_{j \in N_{i}} a_{i j}\left(v_{j}-v_{i}\right) \\
& +h_{i}\left[c_{1}\left(p_{\gamma}-p_{i}\right)+c_{2}\left(v_{\gamma}-v_{i}\right)\right], \quad c_{1}, c_{2}>0,
\end{aligned}
$$

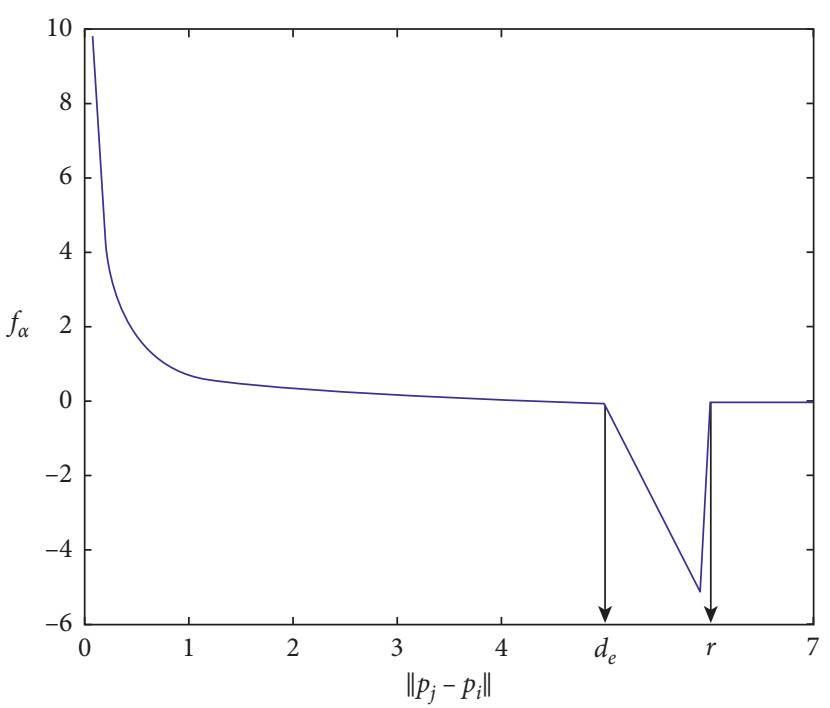

Figure 2: The pairwise action function with a finite cutoff.

where the control indicator $h_{i}$ is utilized to achieve the flocking of multi-agents with partial information exchange. In other words, if the agent $i$ is informed of the group objective, $h_{i}=1$; otherwise, $h_{i}=0$. For example, in Section 6, we assume that there are $M(1 \leq M \leq N)$ informed agents, which means that $h_{i}=1$ for $i=1,2, \ldots, M$, but $h_{i}=0$ for $i=M+1, M+2, \ldots, N$. Note that different from the work of Su et al. [20], we are more focused on selecting an appropriate ratio of the informed agents, which can not only reduce the communication energy consumption, but also enable all the agents (either informed or uninformed) to track the group objective during the flocking process.

\section{Stability Analysis}

As discussed above, an improved flocking algorithm based on the proposed distributed flocking model is proposed. In this section, we first introduce a theorem to establish a stable flocking of multi-agents and then prove this theorem via the Lyapunov stability theorem and LaSalle's invariance principle.

Theorem 4. Consider a group of $N$ mobile agents (or subsystems) with dynamics (10) applying the flocking control protocol (26). Assume that the initial positions and velocities of all agents are chosen at random with the Gaussian distribution. Then, the following statements hold:

(i) No collisions occur between neighbouring agents

(ii) The velocity of all agents asymptotically become consistent

(iii) Flocking of all agents is formed asymptotically

Proof. Define $\widetilde{p}_{i}=p_{i}-p_{\gamma}, \widetilde{v}_{i}=v_{i}-v_{\gamma}$ as the position error vector and velocity error vector, respectively. Consequently, the error dynamic of the agent $i$ is described by 


$$
\begin{aligned}
\widehat{S}_{i}:\left[\begin{array}{c}
\dot{p}_{i} \\
\dot{\widetilde{v}}_{i}
\end{array}\right] & =\left[\begin{array}{ll}
0 & 1 \\
0 & 0
\end{array}\right]\left[\begin{array}{c}
\widetilde{p}_{i} \\
\widetilde{v}_{i}
\end{array}\right]+\left[\begin{array}{l}
0 \\
1
\end{array}\right] u_{i}, \\
{\left[\begin{array}{c}
y_{\widetilde{p}_{i}} \\
y_{\widetilde{v}_{i}}
\end{array}\right] } & =\left[\begin{array}{ll}
1 & 0 \\
0 & 1
\end{array}\right]\left[\begin{array}{c}
\widetilde{p}_{i} \\
\widetilde{v}_{i}
\end{array}\right], \quad i=1,2, \ldots, N .
\end{aligned}
$$

Moreover, let $p_{i j}=p_{i}-p_{j}$ and $\widetilde{p}_{i j}=\widetilde{p}_{i}-\widetilde{p}_{j}$; clearly, $\tilde{p}_{i j}=p_{i j}$. Hence, the collective potential function $V_{i}$ in [7] is modified as

$$
\begin{aligned}
\tilde{V}_{i}\left(\tilde{p}_{i j}\right) & =\sum_{j \in v /\{i\}} F_{\alpha}\left(\left\|\tilde{p}_{i j}\right\|\right) \\
& =\sum_{j \notin N_{i}, j \neq i} F_{\alpha}(r)+\sum_{j \in N_{i}} F_{\alpha}\left(\left\|\tilde{p}_{i j}\right\|\right) .
\end{aligned}
$$

Similarly, flocking control protocol (26) of the agent $i$ can be rewritten as

$$
\begin{array}{r}
u_{i}=\sum_{j \in N_{i}} f_{\alpha}\left(\left\|\tilde{p}_{i j}\right\|\right) e_{i j}+\sum_{j \in N_{i}} a_{i j}\left(\widetilde{v}_{j}-\widetilde{v}_{i}\right)-h_{i}\left[c_{1} \widetilde{p}_{i}+c_{2} \widetilde{v}_{i}\right], \\
c_{1}, c_{2}>0 .
\end{array}
$$

We choose an energy-like Lyapunov function as follows:

$$
Q(\widetilde{p}, \widetilde{v})=\frac{1}{2} \sum_{i=1}^{N}\left(U_{i}(\widetilde{p})+\widetilde{v}_{i}^{T} \widetilde{v}_{i}\right)
$$

where

$$
\begin{aligned}
U_{i}(\widetilde{p}) & =\sum_{j=1, j \neq i}^{N} F_{\alpha}\left(\left\|\widetilde{p}_{i j}\right\|\right)+h_{i} c_{1} \widetilde{v}_{i}^{T} \widetilde{v}_{i} \\
& =\widetilde{V}_{i}\left(\widetilde{p}_{i j}\right)+h_{i} c_{1} \widetilde{v}_{i}^{T} \widetilde{v}_{i}, \\
\widetilde{p} & =\left[\widetilde{p}_{1}, \widetilde{p}_{2}, \ldots, \widetilde{p}_{N}\right]^{T}, \\
\widetilde{v} & =\left[\widetilde{v}_{1}, \widetilde{v}_{2}, \ldots, \widetilde{v}_{N}\right]^{T} .
\end{aligned}
$$

Thanks to the symmetry of $F_{\alpha}(x)$ and the adjacent matrix $A_{a}$ in Definition 2, it follows that

$$
\frac{\partial F_{\alpha}}{\partial \widetilde{p}_{i j}}=\frac{\partial F_{\alpha}}{\partial \tilde{p}_{i}}=-\frac{\partial F_{\alpha}}{\partial \tilde{p}_{j}} .
$$

Then,

$$
\frac{1}{2} \sum_{i=1}^{N} \dot{U}_{i}(\widetilde{p})=\sum_{i=1}^{N}\left(\widetilde{v}_{i}^{T} \nabla_{\tilde{v}_{i}} \widetilde{V}_{i}\left(\widetilde{p}_{i j}\right)+h_{i} c_{1} \widetilde{v}_{i}^{T} \widetilde{p}_{i}\right) .
$$

Consequently,

$$
\begin{aligned}
\dot{Q}(\widetilde{p}, \widetilde{v}) & =\frac{1}{2} \sum_{i=1}^{N} \dot{U}_{i}(\widetilde{p})+\sum_{i=1}^{N} \widetilde{v}_{i}^{T} \dot{\vec{v}}_{i} \\
& =-\widetilde{v}^{T}\left[\left(L(t)+c_{2} H(t)\right) \otimes I_{n}\right] \widetilde{v} \leq 0,
\end{aligned}
$$

where $\otimes$ is the Kronecker product notation, $L(t)$ denotes the Laplacian matrix in Definition $3, H(t)=\operatorname{diag}\left(h_{1}\right.$, $\left.h_{2}, \ldots, h_{N}\right)$, and $I_{n}$ is the identity matrix with $n$ dimensions.

Note that the graph of the adjacent matrix $A_{a}$ is connected, as shown in Figure 1(a). At this moment, $L(t)$ and $H(t)$ are both positive semidefinite matrices, and especially, it is clear that $L(t)+c_{2} H(t)$ is positive semidefinite as well. Hence, $\dot{Q}(\widetilde{p}, \widetilde{v}) \leq 0$, which indicates that $Q(\tilde{p}, \widetilde{v})=Q(t)$ is a nonincreasing function over time $t$. Thereby, $Q(t) \leq Q_{0}$ for all $t \geq 0$, and $Q_{0}$ is the initial value of $Q(t)$. From equations (30) and (31), we conclude that $c_{1} \tilde{p}_{i}^{T} \tilde{p}_{i} \leq 2 Q_{0}$ for any agent $i$, which guarantees the flocking of multi-agents. Therefore, part (iii) is proven.

Since $Q(t)>0$ and $\dot{Q}(t) \leq 0$, we assume that $\Omega=\left\{\left(\tilde{p}^{T}, \widetilde{v}^{T}\right)^{T} \mid Q(t) \leq Q_{0}\right\}$ is an invariant set. From LaSalle's invariance principle, the trajectories of all agents starting from $\Omega$ will converge to the largest set $\Psi=\left\{\left(\widetilde{p}^{T}, \widetilde{v}^{T}\right)^{T} \mid \dot{Q}(t)=0\right\}$. Suppose that the number of connected subgraphs is $m(t)$. For any $t>0$, there is an orthogonal permutation matrix $P(t)$ such that $L(t)+c_{2} H(t)$ can be transformed into the following form:

$$
\begin{aligned}
\widetilde{L}(t)+c_{2} \widetilde{H}(t)= & P(t)\left(L(t)+c_{2} H(t)\right) P^{T}(t) \\
= & \operatorname{diag}\left(L_{1}(t)+c_{2} H_{1}(t), \ldots, L_{k}(t)\right. \\
& \left.+c_{2} H_{k}(t), \ldots, L_{m}(t)+c_{2} H_{m}(t)\right),
\end{aligned}
$$

where $L_{k}(t)$ and $H_{k}(t)$ are the Laplacian matrix and the diagonal matrix corresponding to the $k$ th connected subgraph, respectively, $k=1,2, \ldots, m(t)$. The velocity error vector can also be rewritten as $\widetilde{v}^{p}=\left(P(t) \otimes I_{n}\right) \widetilde{v}$, consequently, we have

$$
\widetilde{v}^{T}\left[\left(L(t)+c_{2} H(t)\right) \otimes I_{n}\right] \widetilde{v}=\left(\widetilde{v}^{p}\right)^{T}\left[\left(\widetilde{L}(t)+c_{2} \widetilde{H}(t)\right) \otimes I_{n}\right] \widetilde{v}^{p} .
$$

As mentioned above, $\widetilde{L}(t)+c_{2} \widetilde{H}(t)$ is positive semidefinite as well. Given (34) and (36), we conclude that $\dot{Q}(t)=$ 0 if and only if $\left(\widetilde{v}^{p}\right)^{T}\left(\widetilde{L}(t) \otimes I_{n}\right) \widetilde{v}^{p}=0$ and $\left(\widetilde{v}^{p}\right)^{T}$ $\left(c_{2} \widetilde{H}(t) \otimes I_{n}\right) \widetilde{v}^{p}=0$, which is equivalent to $v_{1} \equiv \cdots \equiv$ $v_{k} \equiv \cdots \equiv v_{N}$. Therefore, part (ii) is proven.

Finally, we prove part (i) by contradiction. Suppose that at least two agents are colliding during the flocking process; then, we acquire

$$
\begin{aligned}
Q(\widetilde{p}, \widetilde{v}) & =\frac{1}{2} \sum_{i=1}^{N}\left(U_{i}(\widetilde{p})+\widetilde{v}_{i}^{T} \widetilde{v}_{i}\right) \\
& =\frac{1}{2} \sum_{i=1}^{N}\left(\widetilde{V}_{i}\left(\widetilde{p}_{i j}\right)+h_{i} c_{1} \widetilde{v}_{i}^{T} \widetilde{v}_{i}\right)+\frac{1}{2} \sum_{i=1}^{N} \widetilde{v}_{i}^{T} \widetilde{v}_{i} \\
& \geq \frac{1}{2} \sum_{i=1}^{N} \widetilde{V}_{i}\left(\widetilde{p}_{i j}\right) \geq Q_{0},
\end{aligned}
$$

which contradicts the condition $Q(t) \leq Q_{0}$, and this hypothesis is not valid. Therefore, part (i) is proven.

\section{Simulation Results and Discussion}

In this section, examples of numerical simulation are provided to validate the effectiveness of the improved flocking algorithm proposed in Section 4.

Consider a group of 150 agents moving in a two-dimensional space. The initial positions of the agents are randomly distributed in the box $[-50,50] \times[-50,50]$, as 


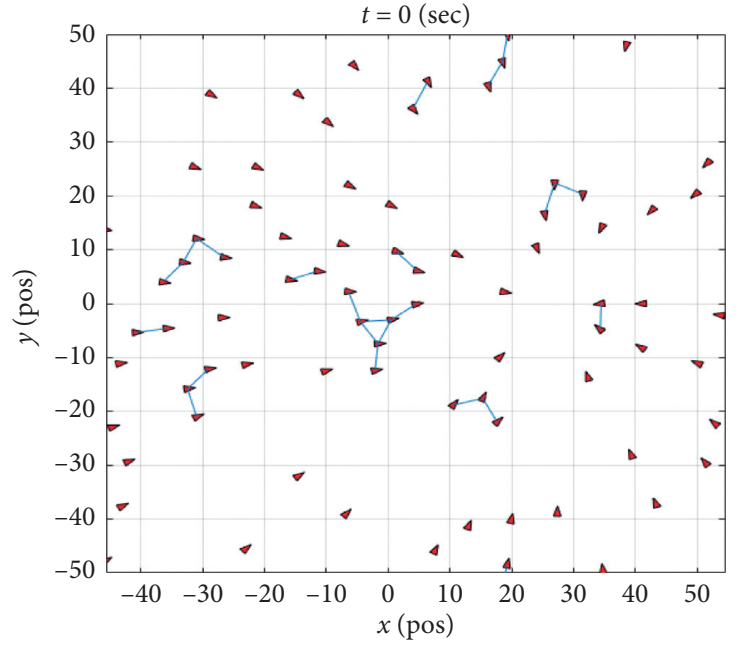

(a)

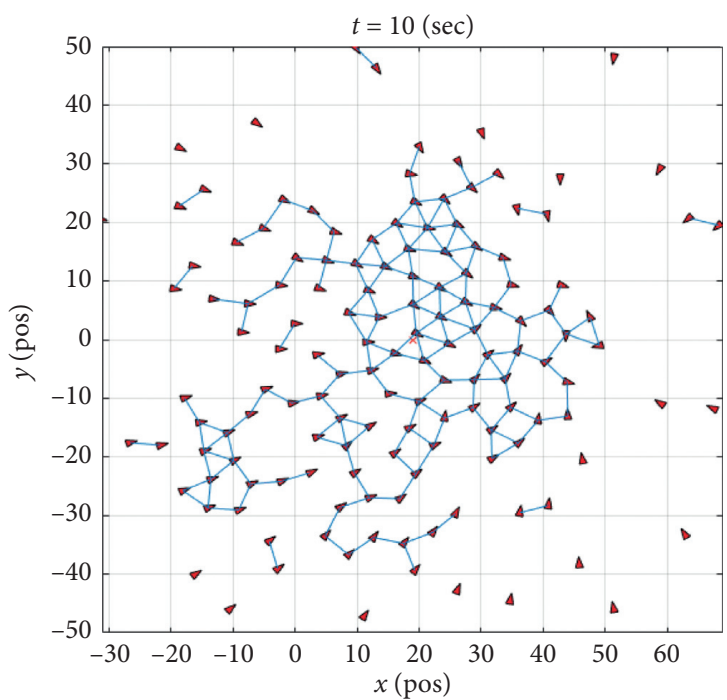

(c)

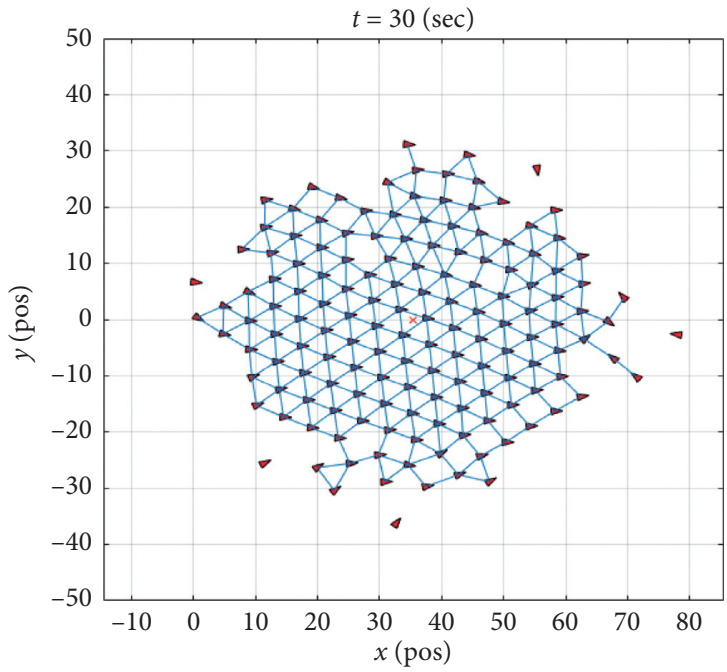

(e)

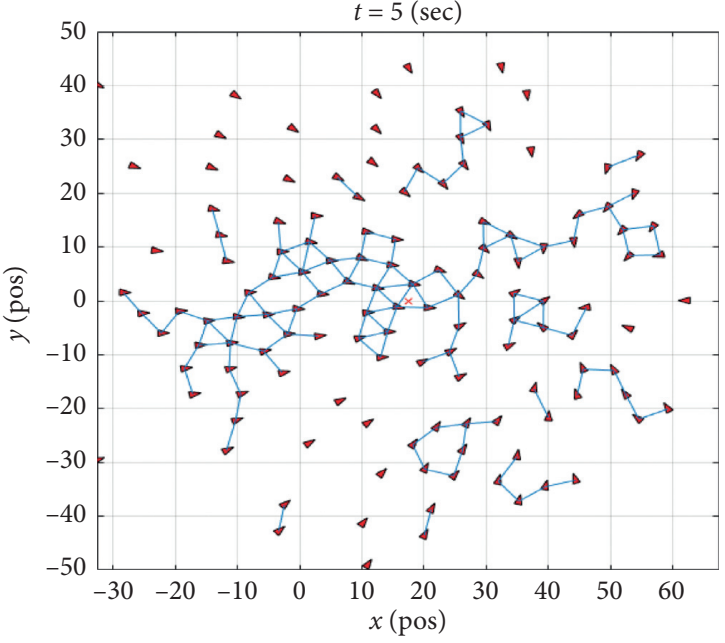

(b)

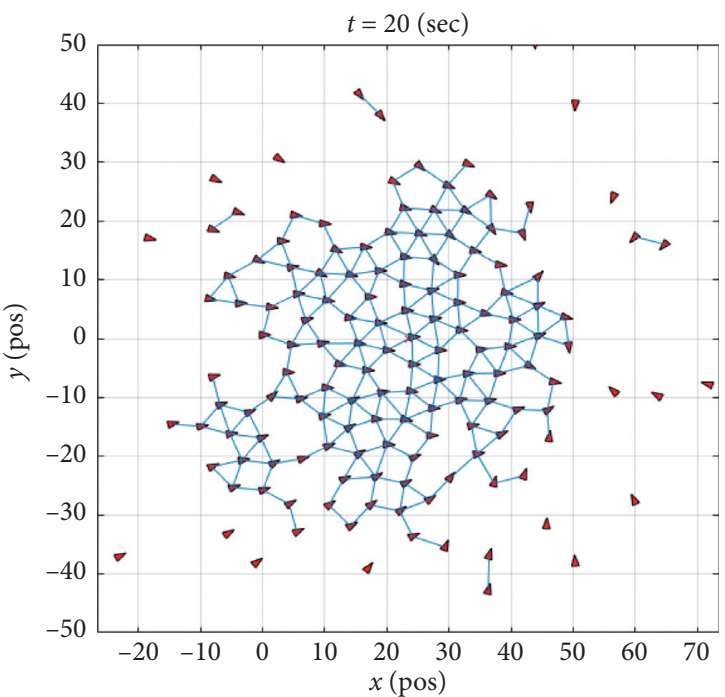

(d)

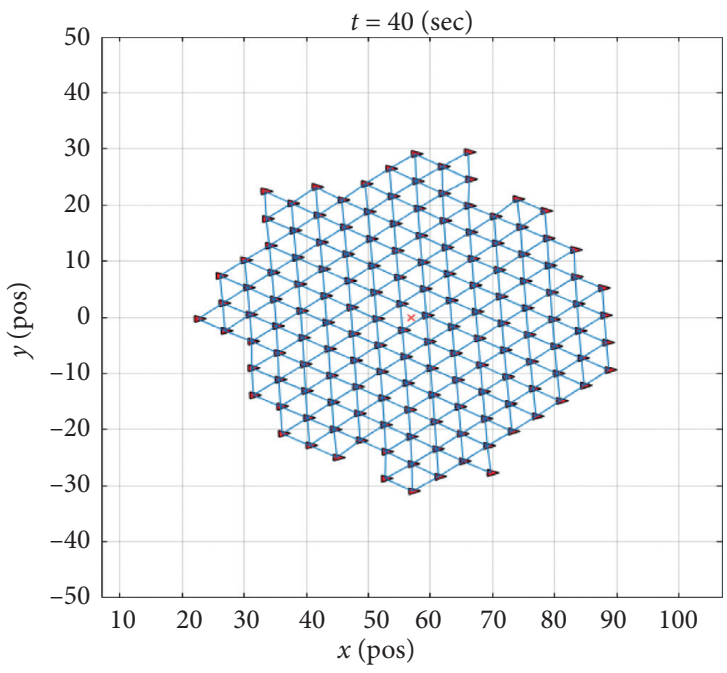

(f)

FIgURE 3: The flocking for $N=150$ agents applying control protocol (26) in 2D. The red triangles denote the positions of all agents; the heading angle of each triangle represents the velocity direction of the corresponding agent, whereas the red $\times$ denotes the position of the group objective. An undirected edge connecting two agents means that they are neighbors of each other. (a) $t=0 \mathrm{sec}$. (b) $t=5 \mathrm{sec}$. (c) $t=10 \mathrm{sec}$. (d) $t=20 \mathrm{sec}$. (e) $t=30 \mathrm{sec}$. (f) $t=40 \mathrm{sec}$. 
TABLE 1: Parameters of the improved flocking algorithm.

\begin{tabular}{lc}
\hline Parameters & Notation and value \\
\hline The equilibrium distance & $d_{e}=5$ \\
The sensing radius & $r=1.2 d_{e}$ \\
Repulsion coefficient of function (24) & $k_{1}=1$ \\
Attraction coefficient of function (24) & $k_{2}=5$ \\
Coefficients of control protocol (26) & $c_{1}=c_{2}=1$ \\
Simulation step size & $\Delta t=0.1 \mathrm{~s}$ \\
\hline
\end{tabular}

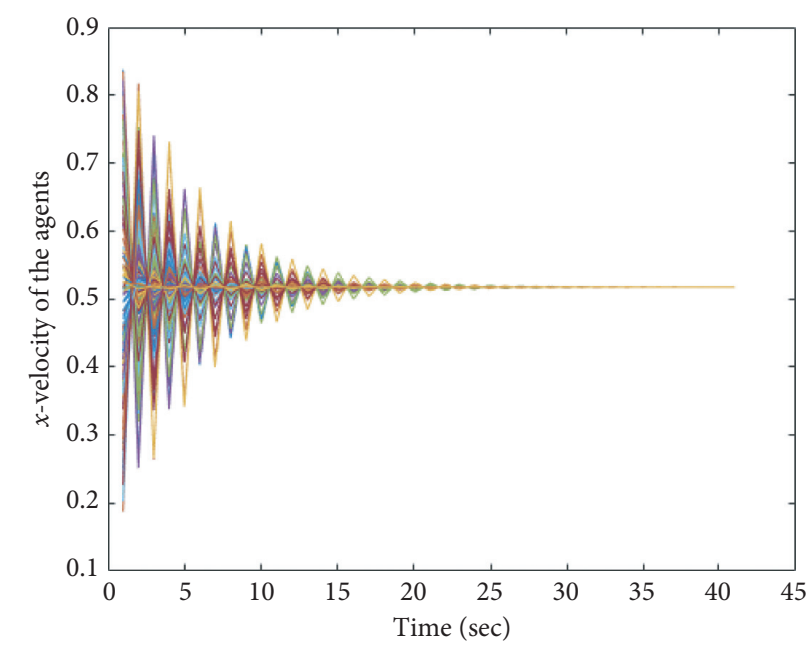

(a)

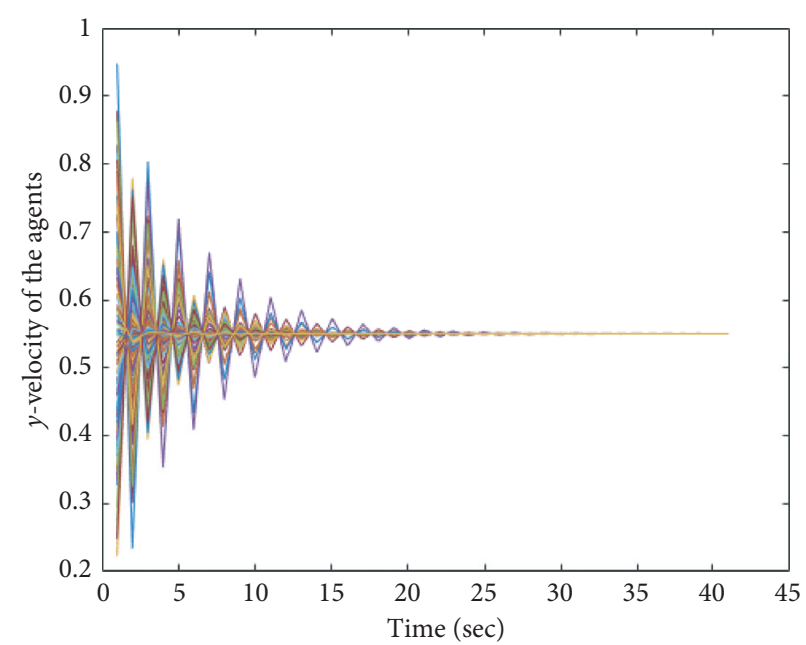

(b)

FIgURE 4: The velocities of all agents during the flocking process. (a) $x$-axis direction. (b) $y$-axis direction.

shown in Figure 3(a). The initial velocities of the agents are randomly chosen from the box $[0,2] \times[0,2]$. The initial position and velocity of the group objective are set as $p_{\gamma}(0)=[0,0]^{T}$ and $v_{\gamma}(0)=[0.5,0]^{T}$, respectively, and marked with a red $\times$. The other parameters are given in Table 1, which remain fixed throughout all simulations. It is worth noting that, for convenience, the above control parameters of the proposed algorithm are all derived from the works of Olfati-Saber [7], Su et al. [20], and Lehn [36]. Furthermore, all simulations are implemented on a platform with the following configurations: $2.20 \mathrm{GHz} \mathrm{CPU}, 8.00 \mathrm{~GB}$ RAM, Windows 10, and MATLAB R2016b.

Figure 3 displays consecutive snapshots of the flocking for $N=150$ agents applying control protocol (26). To decrease the communication energy consumption, only $30 \%$ of the group is randomly selected as the informed agent; hence, there are 45 informed agents. Therefore, the initial state of the group is seen as highly disconnected subgroups, and fragmentation appears in this case, as shown in Figure 3(a). From Figures 3(b) to 3(d), it is clear that more and more agents are connected, but at a certain distance (where no collisions occur). Finally, the group of $N=150$ agents form a geometric flocking, as shown in Figure 3(e), and maintain the formation thereafter, as shown in Figure 3(f).

The velocity consensus during the flocking process is shown in Figure 4. The plot shows that the velocities in the $x$ and $y$-axes eventually converge to a constant set, which means that the velocities of all agents become consistent asymptotically. Therefore, the velocity consensus is achieved.

The above observations are fully consistent with our predictions of Theorem 4, indicated in Section 5.

In the simulation stated above, we assume that all the agents are of equal mass and size and have the same sensing radius $r$ and equilibrium distance $d_{e}$. Nevertheless, for instance, due to the difference in the growth background, social status, and economic strength, etc., it is impossible for two people to be completely identical, in reality. Even for a pair of twins, their cognition and ideology will be different. Therefore, we will fully consider the individual heterogeneity in the following simulations, as we are interested in more than two different species of flocking.

Considering the difference in cognitive level among individuals, we divide the group of $N=150$ agents into three species, whose equilibrium distances $d_{e}$ are set as 5,10, and 15 , respectively. Correspondingly, their sensing radiuses $r$ are set as 6,12 , and 18, respectively (according to the work of Olfati-Saber [7]). The remaining parameters are the same as given in Table 1.

Figure 5 displays consecutive snapshots of the flocking for three species of agents applying the control protocol (26). The initial positions of all agents are randomly distributed in the box $[-70,70] \times[-70,70]$. The initial velocities of all agents are randomly chosen from the box $[-1,1] \times[-1,1]$. The initial position and velocity of the group objective are set 


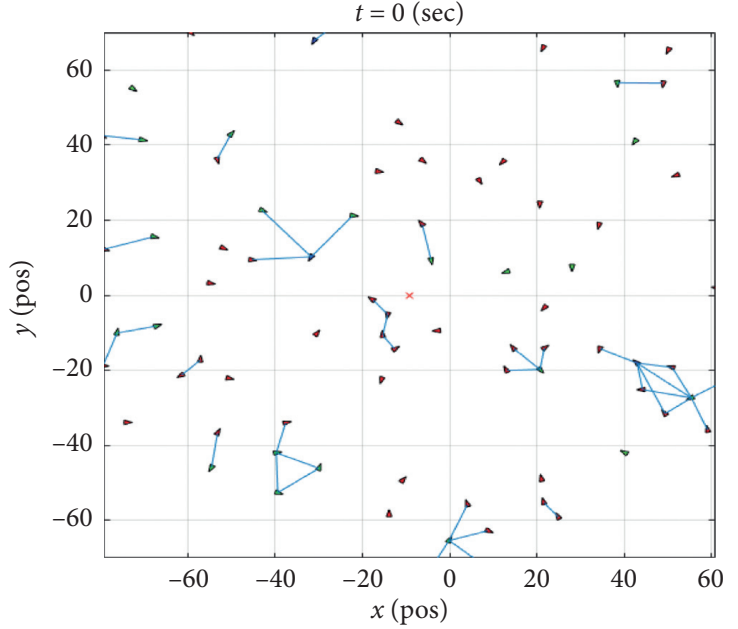

(a)

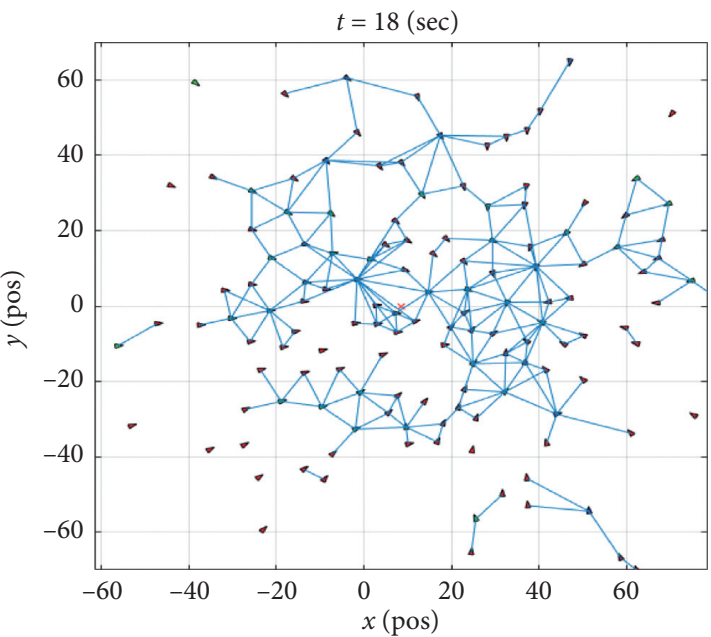

(c)

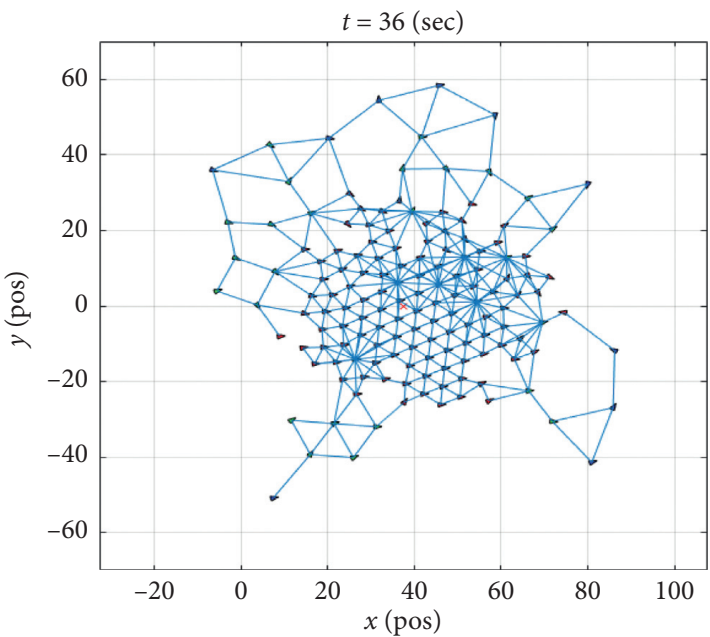

(e)

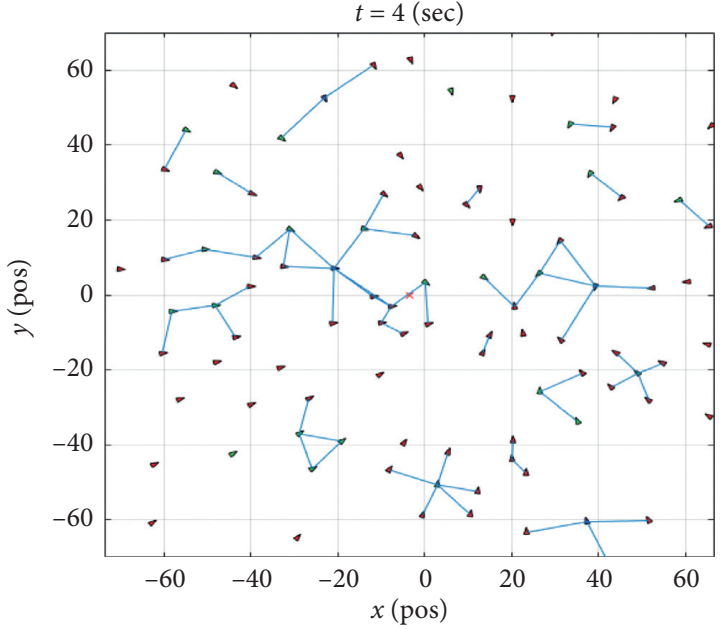

(b)

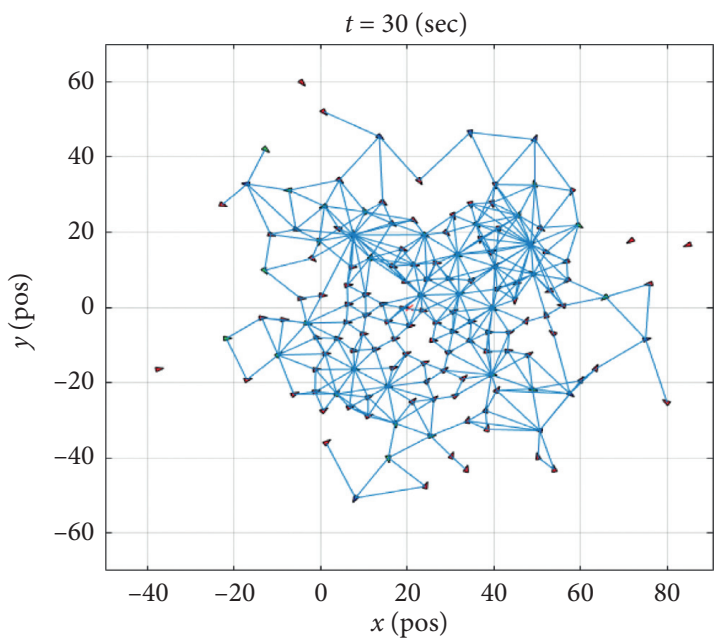

(d)

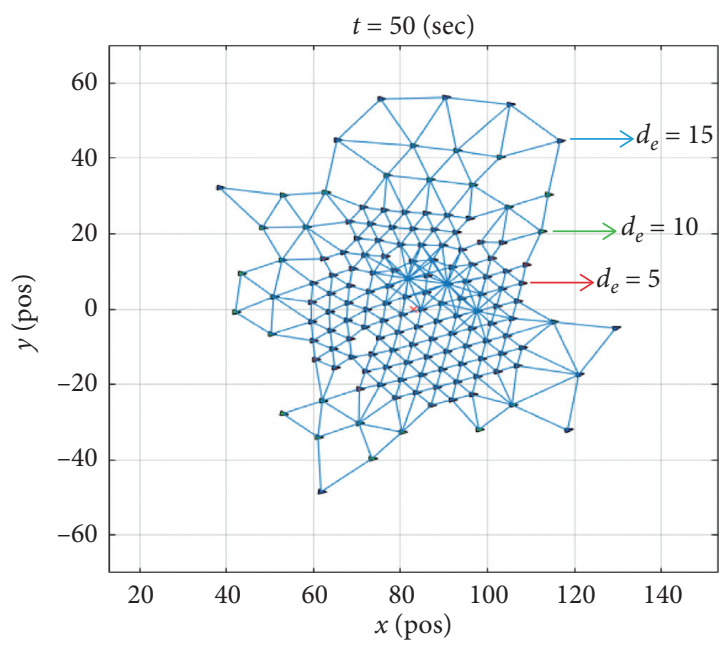

(f)

Figure 5: The flocking for three species of agents applying control protocol (26) in 2D. The triangles denote the positions of all agents; the heading angle of each triangle represents the velocity direction of the corresponding agent, whereas the red $\times$ denotes the position of the group objective. The red, green, and blue triangles represent agents whose equilibrium distance $d_{e}$ is 5,10 , and 15 , respectively. The numbers of red, green, and blue triangles are 110, 30 and 10, respectively. An undirected edge connecting two agents means that they are neighbors of each other. (a) $t=0 \mathrm{sec}$. (b) $t=4 \mathrm{sec}$. (c) $t=18 \mathrm{sec}$. (d) $t=30 \mathrm{sec}$. (e) $t=36 \mathrm{sec}$. (f) $t=50 \mathrm{sec}$. 


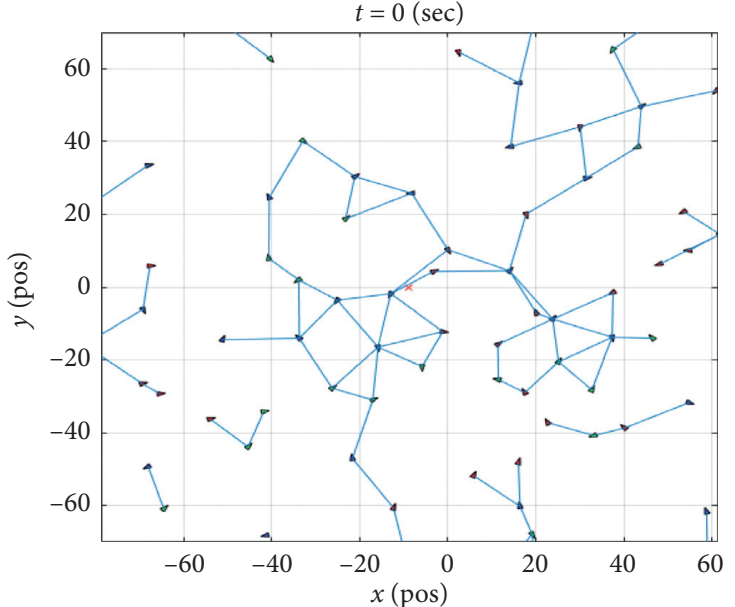

(a)

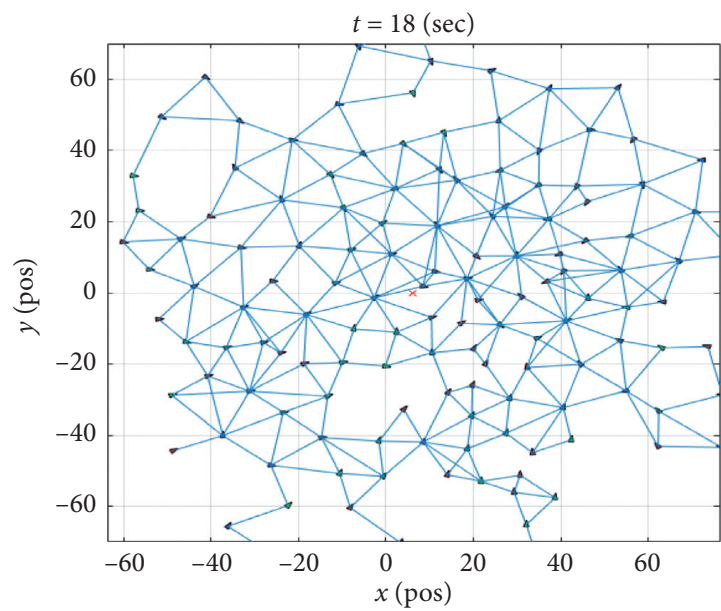

(c)

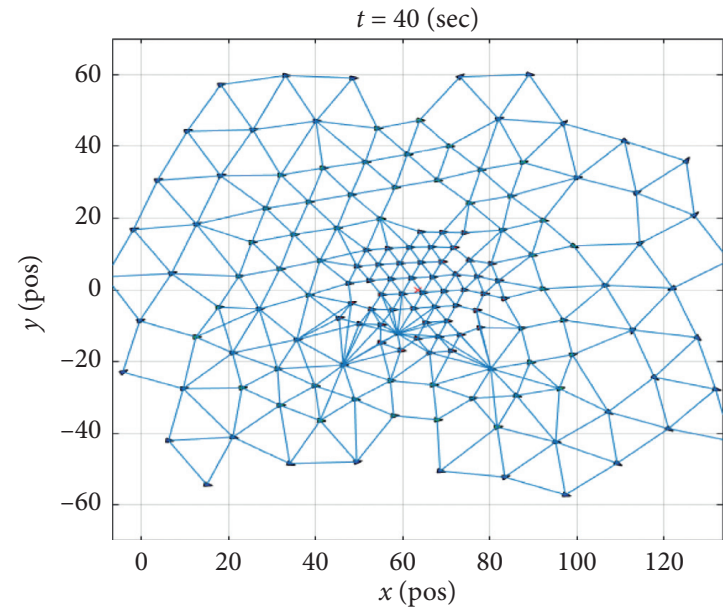

(e)

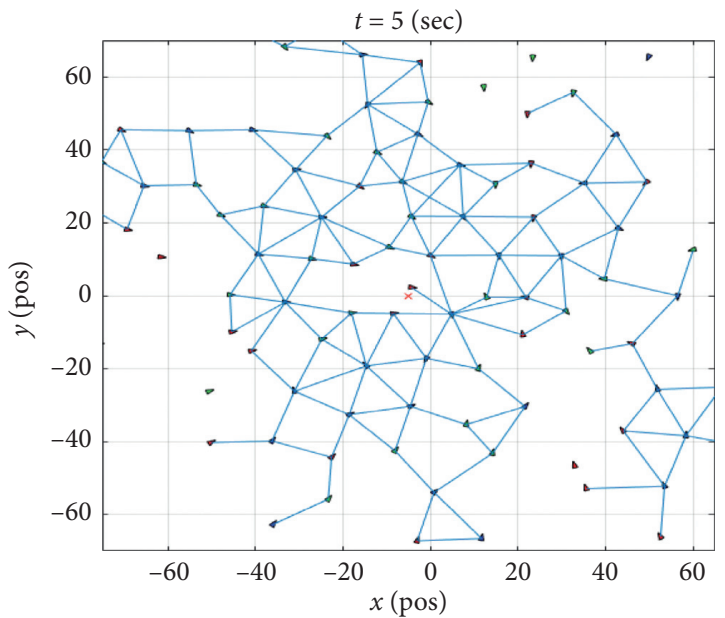

(b)

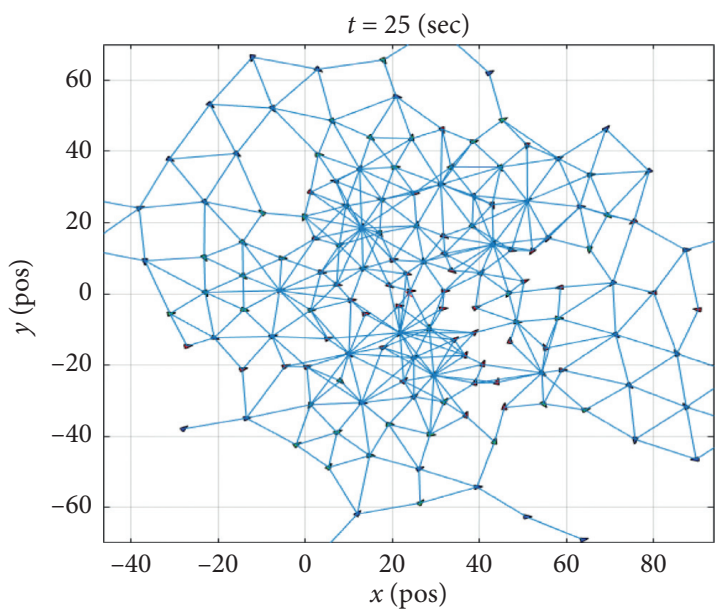

(d)

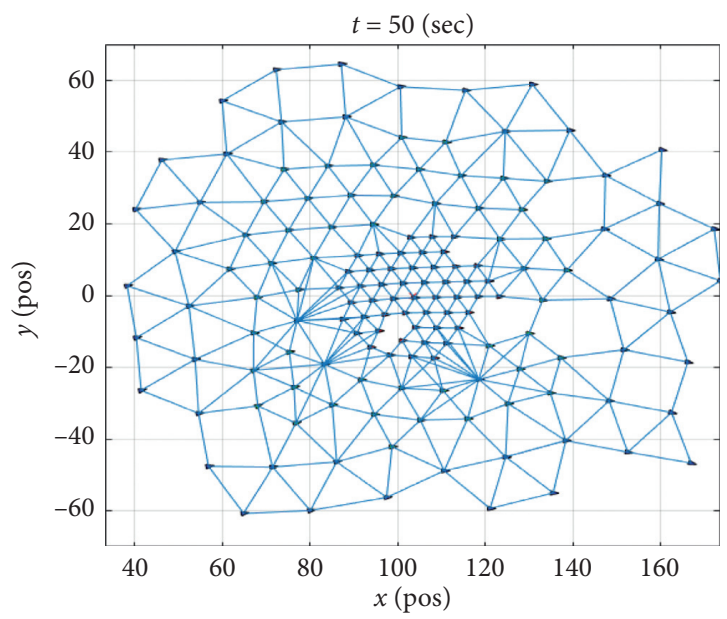

(f)

FIgURE 6: The flocking for three species of agents applying control protocol (26) in 2D. The triangles denote the positions of all agents; the heading angle of each triangle represents the velocity direction of the corresponding agent, whereas the red $\times$ denotes the position of the group objective. The red, green, and blue triangles represent the agent whose equilibrium distance $d_{e}$ is 5,10 , and 15 , respectively, and the number of the three species is equal to 50. An undirected edge connecting two agents means that they are neighbors of each other. (a) $t=0 \mathrm{sec}$. (b) $t=5 \mathrm{sec}$. (c) $t=18 \mathrm{sec}$. (d) $t=25 \mathrm{sec}$. (e) $t=40 \mathrm{sec}$. (f) $t=50 \mathrm{sec}$. 


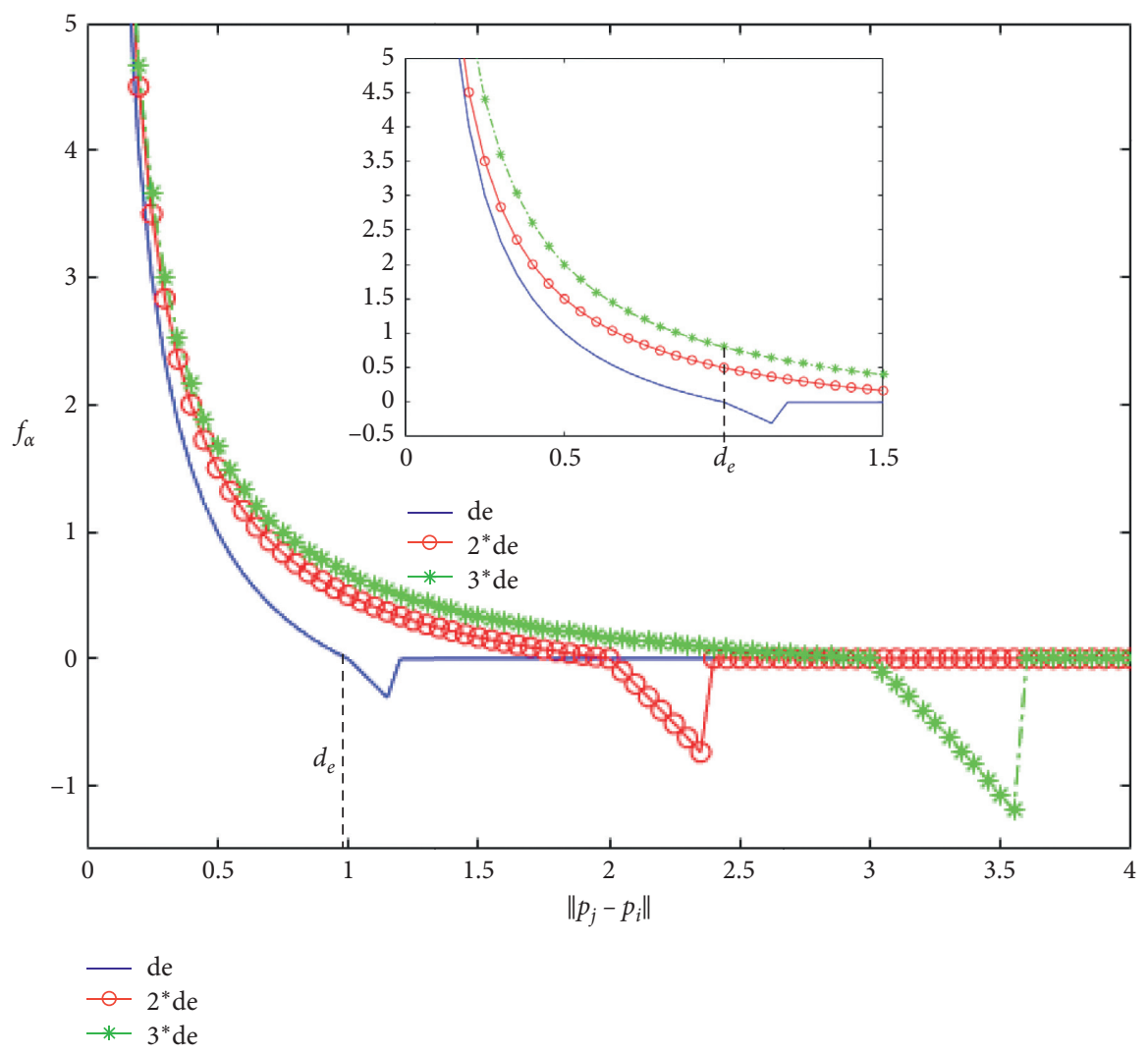

FIgURE 7: The action force function with different equilibrium distances.

as $p_{\gamma}(0)=[-10,0]^{T}$ and $v_{\gamma}(0)=[0.5,0]^{T}, \quad$ respectively. Similarly, there are 45 informed agents. The red, green, and blue triangles represent agents with equilibrium distances $d_{e}$ of 5,10 , and 15 , respectively. The numbers of red, green, and blue triangles are 110, 30, and 10, respectively. Hence, according to the initial state of the group shown in Figure 5(a), the lengths of links between the neighbouring agents are not equal. From Figure 5(b) to 5(e), it is clear that the group of $N=150$ agents eventually form an irregular flocking and maintain the formation thereafter, as can be seen in Figure 5(f). Likewise, no collisions are seen to occur during the flocking process. In this simulation, we find that the green and blue triangles asymptotically move to the periphery and eventually become distributed around the edge of the group, when the multiagent system is stable. Thereafter, the number of each three species is changed to 50 , respectively, while the other parameters remain unchanged. From Figure 6, this self-organizing classification becomes more obvious at the moment. The group is centered on the group objective, and the three species of red, green, and blue agents surround the group objective from the inside to outside. In other words, based on the size of the equilibrium distance $d_{e}$, the three species are arranged surrounding the group objective by $d_{e}$ from small to large. It is interesting to discuss the causes of this phenomenon.

Figure 7 shows the pairwise action function $f_{\alpha}$ with different equilibrium distances. The plot shows that, when the distance between the agent $i$ and the agent $j$ is equal to $d_{e}$, its action force $f_{\alpha}=0$, whereas for the agent $k$ with a larger equilibrium distance (i.e., $2 d_{e}$ and $3 d_{e}$ ), its action force $f_{\alpha}>0$, which can be considered as the repulsive force. Hence, the agent $k$ will gradually move to the edge of the group until its action force is zero. This indicates that the equilibrium distance plays an important role in the flocking. Apparently, a species of agents with a small $d_{e}$ exhibit a stronger cohesive force than the others with the large distance.

It is also worth noting that there are very few agents with large equilibrium distances that are surrounded by agents with small equilibrium distances. At this point, its links appear denser because its sensing radius $r$ is larger than that of the neighbouring agents.

To compare with the typical flocking algorithm [7] and the rigid flocking algorithm based on the IWKCF [25], a simple model is used to calculate the communication energy consumption [38], which is usually applied to wireless microsensor networks. In [38], the radio hardware energy dissipation is related to the distance $d$ between the transmitter and receiver. In other words, for a relatively short distance, the energy dissipation is inversely proportional to $d^{2}$, whereas $d^{4}$ for a relatively long distance. Hence, the communication energy consumption for transmitting a 1-bit message with a distance $d$ is defined as

$$
\begin{aligned}
E_{\mathrm{Tx}}(l, d) & =E_{\mathrm{Tx}-\mathrm{elec}}(l)+E_{\mathrm{Tx}-\mathrm{amp}}(l, d) \\
& = \begin{cases}l E_{\text {elec }}+l \varepsilon_{\mathrm{fs}} d^{2}, & d<d_{\mathrm{c}} \\
l E_{\text {elec }}+l \varepsilon_{\mathrm{mp}} d^{4}, & d \geq d_{\mathrm{c}}\end{cases}
\end{aligned}
$$




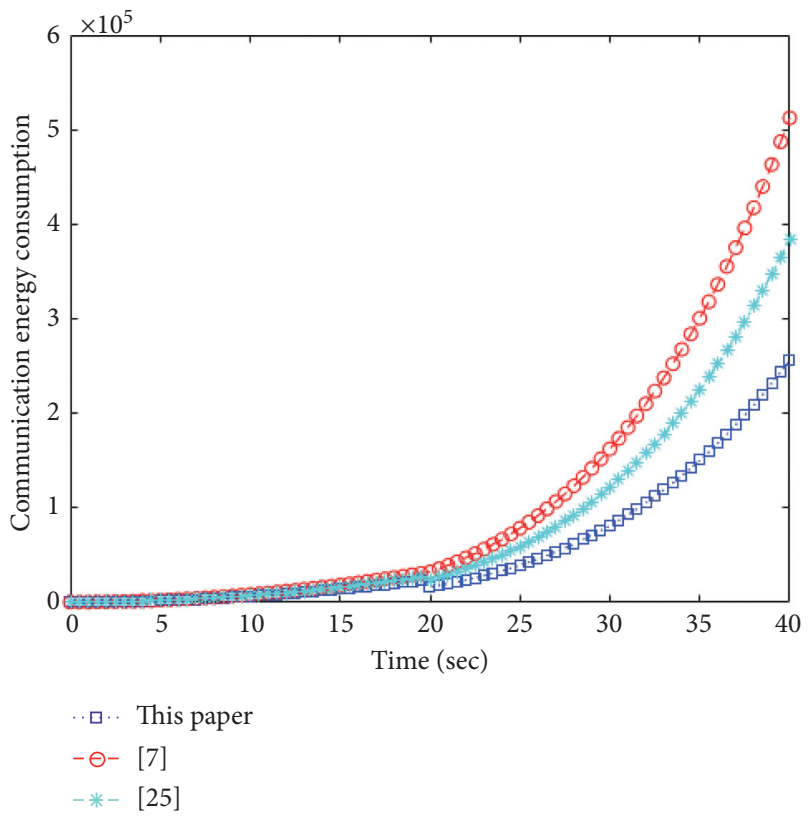

FIgURE 8: Communication energy consumption comparison between the proposed flocking algorithms $[7,25]$.

where $E_{\text {elec }}$ denotes the electronic energy, which depends on factors such as the digital coding, filtering, and spreading of the signal, etc. $\varepsilon_{\mathrm{fs}}$ and $\varepsilon_{\mathrm{mp}}$ are related to the sensitivity of the receiver and the admissible bit-error rate, respectively. $d_{c}$ represents the cross-over distance. For the theoretical experiments in this work, these parameters are set as $E_{\text {elec }}=50 \mathrm{~nJ} / \mathrm{bit}, \quad \varepsilon_{\mathrm{fs}}=10 \mathrm{pJ} / \mathrm{bit} / \mathrm{m}^{2}, \quad d_{\mathrm{c}}=100 \mathrm{~m}, \quad$ and $\varepsilon_{\mathrm{mp}}=0.0013 \mathrm{pJ} / \mathrm{bit} / \mathrm{m}^{2}$, which are the same as the experiments conducted in $[25,38]$.

Figure 8 shows the communication energy consumption compared with the proposed flocking algorithm, the algorithm proposed by Olfati-Saber [7] and Luo et al. [25]. The plot reveals that the communication energy consumption in this paper is significantly lower than that obtained in previous works, which has mainly benefited from the inclusion principle and improved flocking algorithm.

\section{Conclusion}

In this paper, the flocking problem of multi-agents with partial information exchange was investigated based on the inclusion principle. For the purpose of reducing the communication energy consumption, an improved flocking algorithm has been proposed to achieve a stable collective behavior of the multi-agent system. The stability of the multiagent system was analyzed using Theorem 4 and its relevant proof. In addition, considering the individual heterogeneity in both nature and engineering applications, we then investigated the flocking problem of multi-agents with different sensing radiuses and equilibrium distances. Finally, numerical simulations verified the effectiveness of the proposed results. Compared with the above simulation results, we found that there exists an interesting self-organizing classification during the flocking process. It is believed that this phenomenon can provide an insight into the properties of the different species of individual flocking. However, the main limitation of this paper is that the proposed flocking model is only applicable to linear systems. Therefore, future works include, but are not limited to, the following research interests: (1) considering the flocking problem of multi-agents with nonlinear dynamics, (2) applying the proposed flocking algorithm to the formation control of swarm robots.

\section{Data Availability}

The data used to support the findings of this study are available from the corresponding author upon request.

\section{Conflicts of Interest}

The authors declare that there are no conflicts of interest regarding the publication of this paper.

\section{Acknowledgments}

This research was supported by the National Natural Science Foundation of China under Grant nos. 71571091 and 71771112 and University of Science and Technology Liaoning Talent Project under Grant no. 601011507-03.

\section{References}

[1] E. Shaw, "Fish in school," Natural History, vol. 84, no. 8, pp. 40-45, 1975.

[2] H. Ling, G. E. Mclvor, J. Westley et al., "Behavioural plasticity and the transition to order in jackdaw flocks," Nature Communication, vol. 10, no. 1, pp. 1-7, 2019.

[3] H. Ling, G. E. Mclvor, K. Van Der Vaart, R. T. Vaughan, A. Thornton, and N. T. Ouellette, "Costs and benefits of social relationships in the collective motion of bird flocks," Nature Ecology \& Evolution, vol. 3, no. 6, pp. 943-948, 2019.

[4] A. Okubo, "Dynamical aspects of animal grouping: swarms, schools, flocks, and herds," Advances in Biophysics, vol. 22, pp. 1-94, 1986.

[5] X. Guo, J. Lu, A. Alsaedi, and F. E. Alsaadi, "Bipartite consensus for multi-agent systems with antagonistic interactions and communication delays," Physica A: Statistical Mechanics and Its Applications, vol. 495, pp. 488-497, 2018.

[6] Y. Shang, "Group consensus of multi-agent systems with switching topologies and stochastic inputs," Physics Letters A, vol. 377, no. 25-27, pp. 1582-1586, 2013.

[7] R. Olfati-Saber, "Flocking for multi-agent dynamic systems: algorithms and theory," IEEE Transactions on Automatic Control, vol. 51, no. 3, pp. 401-420, 2006.

[8] H.-T. Zhang, Z. Cheng, G. Chen, and C. Li, "Model predictive flocking control for second-order multi-agent systems with input constraints," IEEE Transactions on Circuits and Systems I: Regular Papers, vol. 62, no. 6, pp. 1599-1606, 2015.

[9] C. W. Reynolds, "Flocks, herds and schools: a distributed behavioral model," ACM SIGGRAPH Computer Graphics, vol. 21, no. 4, pp. 25-34, 1987.

[10] Y. He and X. Mu, "Cucker-smale flocking subject to random failure on general digraphs," Automatica, vol. 106, pp. 54-60, 2019.

[11] T. Zuo, H. Min, Q. Tang, and Q. Tao, "A robot SLAM improved by quantum-behaved particles swarm optimization," 
Mathematical Problems in Engineering, vol. 2018, Article ID 1596080, 11 pages, 2018.

[12] X. Wu, Y. Wang, and Y. Hu, "A swarm intelligent algorithm based route maintaining protocol for mobile sink wireless sensor networks," Mathematical Problems in Engineering, vol. 2015, Article ID 823909, 10 pages, 2015.

[13] H. Zhou, W. Zhou, and W. Zeng, "Flocking control of multiple mobile agents with the rules of avoiding collision," Mathematical Problems in Engineering, vol. 2015, Article ID 529538, 9 pages, 2015.

[14] Q. Liu, M. He, D. Xu, N. Ding, and Y. Wang, "A mechanism for recognizing and suppressing the emergent behavior of UAV swarm," Mathematical Problems in Engineering, vol. 2018, Article ID 6734923, 14 pages, 2018.

[15] B. Li, J. Li, and K. Huang, "Modeling and flocking consensus analysis for large-scale UAV swarms," Mathematical Problems in Engineering, vol. 2013, Article ID 368369, 9 pages, 2013.

[16] T. Vicsek, A. Czirók, E. Ben-Jacob, I. Cohen, and O. Shochet, "Novel type of phase transition in a system of self-driven particles," Physical Review Letters, vol. 75, no. 6, pp. 12261229, 1995.

[17] J. Toner and Y. Tu, "Flocks, herds, and schools: a quantitative theory of flocking," Physical Review E, vol. 58, no. 4, pp. 4828-4858, 1998.

[18] D. Helbing, I. Farkas, and T. Vicsek, "Simulating dynamical features of escape panic," Nature, vol. 407, no. 6803, pp. $487-490,2000$.

[19] A. Jadbabaie, J. Jie Lin, and A. S. Morse, "Coordination of groups of mobile autonomous agents using nearest neighbor rules," IEEE Transactions on Automatic Control, vol. 48, no. 6, pp. 988-1001, 2003.

[20] H. Su, X. Wang, and Z. Lin, "Flocking of multi-agents with a virtual leader," IEEE Transactions on Automatic Control, vol. 54, no. 2, pp. 293-307, 2009.

[21] X. Luo, S. Li, and X. Guan, "Flocking algorithm with multitarget tracking for multi-agent systems," Pattern Recognition Letters, vol. 31, no. 9, pp. 800-805, 2010.

[22] H. Pei, S. Chen, and Q. Lai, "A local flocking algorithm of multi-agent dynamic systems," International Journal of Control, vol. 88, no. 11, pp. 2242-2249, 2015.

[23] J. Xi, Z. Shi, and Y. Zhong, "Output consensus analysis and design for high-order linear swarm systems: partial stability method," Automatica, vol. 48, no. 9, pp. 2335-2343, 2012.

[24] Y. Cao and W. Ren, "Distributed coordinated tracking with reduced interaction via a variable structure approach," IEEE Transactions on Automatic Control, vol. 57, no. 1, pp. 33-48, 2012.

[25] X. Luo, X. Li, S. Li, Z. Jiang, and X. Guan, "Flocking for multiagent systems with optimally rigid topology based on information weighted Kalman consensus filter," International Journal of Control, Automation and Systems, vol. 15, no. 1, pp. 138-148, 2016.

[26] S. Chen, H. Pei, Q. Lai, and H. Yan, "Multitarget tracking control for coupled heterogeneous inertial agents systems based on flocking behavior," IEEE Transactions on Systems, Man, and Cybernetics: Systems, vol. 49, no. 12, pp. 2605-2611, 2019.

[27] H. Zhang, J. Zhang, G.-H. Yang, and Y. Luo, "Leader-based optimal coordination control for the consensus problem of multiagent differential games via fuzzy adaptive dynamic programming," IEEE Transactions on Fuzzy Systems, vol. 23, no. 1, pp. 152-163, 2015.

[28] H. G. Tanner, "Flocking with obstacle avoidance in switching networks of interconnected vehicles," in Proceedings of the
IEEE International Conference Robotics Automatic, vol. 3, pp. 3006-3011, New Orleans, LA, USA, May 2004.

[29] W. Ren and E. Atkins, "Distributed multi-vehicle coordinated control via local information exchange," International Journal of Robust and Nonlinear Control, vol. 17, no. 10-11, pp. 1002-1033, 2006.

[30] X.-B. Chen and S. S. Stanković, "Decomposition and decentralized control of systems with multi-overlapping structure," Automatica, vol. 41, no. 10, pp. 1765-1772, 2005.

[31] X.-B. Chen and S. S. Stankovic, "Overlapping decentralized approach to automation generation control of multi-area power systems," International Journal of Control, vol. 80, no. 3, pp. 386-402, 2007.

[32] X.-B. Chen, W.-B. Xu, T.-Y. Huang, X.-Y. Ouyang, and S. S. Stankovic, "Pair-wise decomposition and coordinated control of complex systems," Information Sciences, vol. 185, no. 1, pp. 78-99, 2012.

[33] D. M. Stipanović, G. K. Inalhan, and R. Teo, "Decentralized overlapping control of a formation of unmanned aerial vehicles," Automatica, vol. 40, no. 8, pp. 1285-1296, 2004.

[34] A. Aybar, "Decentralized controller design for forbidden states avoidance in timed discrete event systems," International Journal of Computers Communications \& Control, vol. 5, no. 4, pp. 447-457, 2010.

[35] W. W. Wood and F. R. Parker, "Monte Carlo equation of state of molecules interacting with the Lennard-Jones potential. I. A supercritical isotherm at about twice the critical temperature," Journal of Chemical. Physics, vol. 27, no. 3, pp. 720-733, 2004.

[36] J.-M. Lehn, "Toward self-organization and complex matter," Science, vol. 295, no. 5564, pp. 2400-2403, 2002.

[37] O. Mason and M. Verwoerd, "Graph theory and networks in biology," IET Systems Biology, vol. 1, no. 2, pp. 89-119, 2007.

[38] W. B. Heinzelman, A. P. Chandrakasan, and H. Balakrishnan, "An application-specific protocol architecture for wireless microsensor networks," IEEE Transactions on Wireless Communications, vol. 1, no. 4, pp. 660-670, 2002. 